\title{
PERSPECTIVE Digital phenotyping approaches and mobile devices enhance CNS biopharmaceutical research and development
}

\author{
Daniel G. Smith ${ }^{1}$ \\ Neuropsychopharmacology (2018) 43:2504-2505; https://doi.org/10.1038/s41386-018-0222-6
}

As goes the omnipresence of smartphones and wearable biobehavioral technologies, such as activity tracking wrist bands, so goes the integration of these technologies into the lives of individuals with central nervous system (CNS) disorders and into the arena of clinical brain and behavior research [1-5]. Digital phenotyping is one result of the interaction between pervasive personal technology, patients and neurobehavioral researchers and drug developers. Defined as the momentary, ecological quantification of behavior, as measured using sensors embedded in personal technologies [6] and coupled with digitized symptom rating scales and behavioral tasks, digital phenotyping is about unobtrusively capturing the patterns by which individuals interact with their devices and the environment.

Advertisers and others already know that "Where you go is who you are." [7]. It is valuable insight and it is largely dependent on one of many possible smartphone and wearable device data streams (geolocation). The picture gets significantly richer by integrating other data types and related metadata [8-10]. Talk, text, and email communication and social media app usage reveal social behavioral patterns and linguistic capacity, sleep and wake cycle data can be derived from around-the-clock phone interaction patterns, like dips and spikes in screen touches or acceleration (movement), and typing speed data may be a clinically-meaningful surrogate of psychomotor function. These findings suggest that digital phenotyping is a vivid, scalable rainbow with a pot of human behavioral gold at the end.

Researchers are picking these data types apart and revealing their promise, limitations and, most importantly, their relevance to patient experience and the understanding and treatment of neurobehavioral disorders $[11,12]$. One overarching vision is that these data streams, when collected and analyzed using rigorous scientific and statistical methods by trained behavioral specialists, will illuminate a "real-world" picture of patients' lives and the trajectory of disease at a multi-faceted, individual-subject level. In a clinical trial setting, the intermittent, busy, and sometimes long per protocol visits to a medical facility are artificial and merely snapshots of a patients' condition.

Validated digital phenotyping techniques have the potential to add objective, multi-point and between-visit data that complement data collected during site visits. Therein lays the value proposition for digital phenotyping to biopharmaceutical companies invested in neurobehavioral research and development. Could increasing the availability or acceptance of mobile devices and digital phenotyping approaches provide more objective metrics that enhance insight on safety, efficacy, approvability, and long-term outcomes in the course of developing therapeutics? Could they increase patient pools and enhance recruitment for clinical trials by enabling remote versus in-clinic data collection? Is it possible that they reduce the cost of clinical trials by increasing power (through high frequency data collection) in fewer participants? Can it be done without sacrificing quality of care or increasing morbidity?

An evidence basis that addresses those questions in the affirmative is necessary to evolve beyond the prototypical model of interventional clinical trials for neurobehavioral disorders. That model relies on short treatment durations (8-12 weeks), intermittent "snapshots-in-time" site visits and a single subjectivelyrated primary endpoint in groups of patients with unaccountedfor heterogeneity. It has many sources of significant risk and has a well-documented high rate of failure [13]. Those features and patients' positive expectancy about receiving active drug, a deeper rapport with clinicians and an abnormally high level of attention from medical professionals confound the model.

There is no single solution to these issues. However, as part of a multi-modal approach that integrates clinical, behavioral and neurophysiological patient data, scientifically validated digital phenotyping approaches bring longitudinal read-outs captured outside of the lab that circumvent some issues, like reliance on snapshots and subjective ratings. To that end, digital data streams are being contextualized within innovative re-conceptualizations of brain-behavior diseases that integrate clinical, behavioral, neurophysiological, molecular, and genetic information [14]. The deepened insight into neurobehavioral disease trajectory and treatment could challenge the categorical nosology of neurobehavioral disorders like other quantitative, multi-modal research [15-17].

Innovative experiments are underway that explore the value of cutting-edge digital biomarker technologies in biopharmaceutical clinical research and trials $[18,19]$. A critical goal of this work is to demonstrate whether these data types are clinically meaningful, e.g., that they predict or otherwise relate to clinical signs and symptoms and can support internal decision-making or regulatory objectives for developing new medicines. Some research suggests that digital phenotyping endpoints have predictive potential for medical events, like adverse responses to drugs during clinical trials or relapse in patients with substance use disorder or psychosis [20].

Do smartphone geolocation and other data streams, coupled with digitized patient-reported outcomes (in the form of surveys or behavioral tasks) and intermittent clinical interviews improve diagnoses or patient stratification? Do they detect signs of efficacy that are missed during structured, intermittent site visits or that

${ }^{1}$ Department of Clinical Research, Alkermes Incorporated, Waltham, MA 02451, USA

Correspondence: Daniel G. Smith (dsmith@alkermes.com)

Received: 10 August 2018 Revised: 6 September 2018 Accepted: 11 September 2018

Published online: 18 September 2018 
are indicative of long-term functional improvements and greater quality of life? There are more questions than answers on the near-term horizon about the value of digital phenotyping broadly, and its role in improving clinical trials specifically. However, it is certain that personal digital technologies need to be put to the test in rigorous studies to define their value to researchers, developers, and patients.

Another certainty is that no digital phenotyping research will be worthwhile unless rigorous and transparent clinical and data sciences methods are used to generate and analyze the data, or if patient data privacy or the integrity of electronic data systems are compromised. Additional factors that challenge the usefulness of digital phenotyping data include seemingly small things, like a patient that occasionally shares their phone with another person. It includes common issues, like dealing with data missingness or misleading data from patients that have multiple phones or SIM cards and generate distorted data sets. And, there are fatal issues, like failing to establish data access permissions, an audit trail, and processes to ensure the security and technical validity of electronic systems for capturing, transferring, and storing data.

Even before starting a digital phenotyping endeavor, it can be daunting to choose the best fit-for-purpose technologies. For example, there are myriad wearables but they are generally noninteroperable and have different strengths and limitations. There are user-friendly software and analytical solutions (e.g., ResearchKit for iOS or ResearchStack for Android) but they only deliver summaries of the underlying data. Much greater sophistication is required to collect the (more valuable) raw smartphone data and to develop the necessary data analysis and modeling tools to deal with them. Navigating these and other challenges is a major undertaking. In the end, teams that include patients, clinicians, scientists, engineers, technologists and ethicists, and who respect scientific principles, will be the only ones that prove the value of digital phenotyping in research and its potential to enhance clinical drug development.

\section{ADDITIONAL INFORMATION}

Competing interests: The author declares no competing interests.

Publisher's note: Springer Nature remains neutral with regard to jurisdictional claims in published maps and institutional affiliations.

\section{REFERENCES}

1. Torous J, Chan SR, Yee-Marie Tan S, Behrens J, Mathew I, Conrad EJ, et al. Patient smartphone ownership and interest in mobile apps to monitor symptoms of mental health conditions: a survey in four geographically distinct psychiatric clinics. JMIR Ment Health. 2014;1:e5.
2. Torous J, Friedman R, Keshavan M. Smartphone ownership and interest in mobile applications to monitor symptoms of mental health conditions. JMIR Mhealth Uhealth. 2014;2:e2.

3. Gold M, Amatniek J, Carrillo MC, Cedarbaum JM, Hendrix JA, Miller BB, et al. Digital technologies as biomarkers, clinical outcomes assessment, and recruitment tools in Alzheimer's disease clinical trials. Alzheimers Dement. 2018;4:234-42.

4. Espay AJ, Bonato P, Nahab FB, Maetzler W, Dean JM, Klucken J, et al. Technology in Parkinson's disease: challenges and opportunities. Mov Disord. 2016;31:1272-82.

5. Onnela JP, Rauch SL. Harnessing smartphone-based digital phenotyping to enhance behavioral and mental health. Neuropsychopharmacology . 2016;41:1691-6.

6. Torous J, Staples P, Onnela JP. Realizing the potential of mobile mental health: new methods for new data in psychiatry. Curr Psychiatry Rep. 2015;17:602.

7. The Boston Globe. Every step you take. http://www.apps.bostonglobe.com. Accessed 7 July 2018

8. Arroyo-Gallego T, Ledesma-Carbayo MJ, Sanchez-Ferro A, Butterworth I, Mendoza CS, Matarazzo M, et al. Detection of motor impairment in Parkinson's Disease via mobile touchscreen typing. IEEE Trans Biomed Eng. 2017;64:1994-2002.

9. Dagum P.Digital biomarkers of cognitive function. npj Digital Med. 2018;1:10

10. Staples P, Torous J, Barnett I, Carlson K, Sandoval L, Keshavan M, et al. A comparison of passive and active estimates of sleep in a cohort with schizophrenia. NPJ Schizophr. 2017;3:37.

11. Tal A, Torous J. The digital mental health revolution: opportunities and risks. Psychiatr Rehabil J. 2017;40:263-5.

12. Torous J, Staples P, Barnett I, Sandoval LR, Keshavan M, Onnela JP. Characterizing the clinical relevance of digital phenotyping data quality with applications to a cohort with schizophrenia. npj Digit Med. 2018;1:15.

13. Hyman SE. Psychiatric drug development: diagnosing a crisis. Cerebrum. 2013 Apr 2:2013:5.

14. Torous J, Onnela JP, Keshavan M. New dimensions and new tools to realize the potential of RDoC: digital phenotyping via smartphones and connected devices. Transl Psychiatry. 2017;7:e1053.

15. Clementz BA, Sweeney JA, Hamm JP, Ivleva El, Ethridge LE, Pearlson GD, et al. Identification of distinct psychosis biotypes using brain-based biomarkers. Am J Psychiatry. 2016;173:373-84.

16. Drysdale AT, Grosenick L, Downar J, Dunlop K, Mansouri F, Meng Y, et al. Resting state connectivity biomarkers define neurophysiological subtypes of depression. Nat Med. 2017;23:28-38.

17. Williams LM. Precision psychiatry: a neural circuit taxonomy for depression and anxiety. Lancet Psychiatry. 2016;3:472-80.

18. Cambridge Cognition. Cognition Kit wearable technology demonstrates high compliance among patients with major depressive disorder (MDD). http://www. cambridgecognition.com/news/entry/cognition-kit-wearable-demonstrates-highcompliance-among-MDD-patients. Accessed 7 July 2018.

19. Smith DG, Saljooqi K, Alvarez-Horine S, Dagum P, Madrid A. Exploring nove behavioral tasks and digital phenotyping technologies as adjuncts to a clinical trial of BTRX-246040. https://isctm.org/public_access/Feb2018/PDFs/Smithposter.pdf. Accessed 7 July 2018.

20. Barnett I, Torous J, Staples P, Sandoval L, Keshavan M, Onnela JP. Relapse prediction in schizophrenia through digital phenotyping: a pilot study. Neuropsychopharmacology. 2018:43:1660-6. 